\title{
DEFECT RELATIONS FOR DEGENERATE MEROMORPHIC MAPS
}

\author{
WANXI CHEN
}

\begin{abstract}
Using a concept called subgeneral position and adapting a weight function created by E. I. Nochka, this work proves the Cartan's conjecture on defect relations for a degenerate meromorphic map from a parabolic manifold into a projective space.
\end{abstract}

In value distribution theory, defect relations for a nondegenerate meromorphic map are well known. When the map is degenerate, the situation is not so good. A defect relation was conjectured by H. Cartan [1] in 1933. It remained unproved until 1982 when Nochka [4] was published. Nochka [4] deals with only a degenerate meromorphic curve; i.e., the domain of the map has dimension one. When the domain has dimension greater than one, no defect relations for degenerate meromorphic maps have been known. After a discussion of the Second Main Theorem, this work has succeeded in establishing a Nevanlinna defect relation for a meromorphic map (degenerate or nondegenerate) from a parabolic manifold into a projective space. The defect relation is rather general. It contains as special cases all the known Nevanlinna defect relations for such kind of meromorphic maps.

The basic idea of this work is very simple: transform the problem with a degenerate meromorphic map into the one with a nondegenerate meromorphic map. The difficulty is, after such a transformation, the originally given hyperplanes in general position become hyperplanes not in general position. We overcome this obstacle by introducing a concept called subgeneral position (see Chen $[2,3])$.

The defect relation comes from the Second Main Theorem, while the latter depends on two inequalities: the Product to Sum Estimate and the Ahlfors Estimate. Degeneracy of a map does not affect the Ahlfors Estimate, but it does affect the Product to Sum Estimate. To build a desired Product to Sum Estimate, we adapt a weight function created by Nochka [4] and proved in Nochka [5].

To save pages and to make basic ideas clearer, we take two steps: first, we prove only a result for hyperplane targets, the defect relations for associated

Received by the editors September 21, 1987.

1980 Mathematics Subject Classification (1985 Revision). Primary 32A22, 32H30.

Key words and phrases. Value distribution, defect relations, degenerate, subgeneral position.

This article is partially supported by NSF grant \#USE-8851944. 
maps are referred to Chen [2]; second, we make the preliminaries for this work very brief. Some basic concepts are used in this work without definition. The details of them can be found in Stoll [6]. Part A of Stoll [7] is also good enough.

\section{Preliminaries}

Let $V$ be a complex vector space of dimension $k+1$ with $1 \leq k<\infty$. Let $V^{*}$ be the dual vector space. Denote $\mathbb{Z}[a, b]=\{x \mid x$ is an integer, $a \leq x \leq b\}$ and $Q=\mathbb{Z}[1, q]$. Let $\left\{\alpha_{j}\right\}_{j \in Q}$ be a family of vectors in $V^{*}-\{0\}$ and $a_{j}=\mathbb{P}\left(\alpha_{j}\right) \in \mathbb{P}\left(V^{*}\right)$. If $P \subseteq Q$, then denote $H(P)=\operatorname{span}\left\{\alpha_{j} \mid j \in P\right\}$ and $d(P)=\operatorname{dim} H(P)$. Suppose the integers $k, n$ and $q$ satisfy $n \geq k \geq 1$ and $2 n-k+1<q<\infty$.

Definition 0.1 (Chen $[2,3]) .\left\{\alpha_{j}\right\}_{j \in Q}$ are called in $n$-subgeneral position if any $n+1$ of them generate $V^{*} .\left\{a_{j}\right\}_{j \in Q}$ are called in $n$-subgeneral position iff $\left\{\alpha_{j}\right\}_{j \in Q}$ are in $n$-subgeneral position.

Lemma 0.2 (Chen $[2,3]$ ). Suppose $l \in \mathbb{Z}[1, q],\left\{\alpha_{j}\right\}_{j \in Q}$ are in $n$-subgeneral position and $\lambda: \mathbb{Z}[1, l] \rightarrow \mathbb{Z}[1, q]$ is an injective map. Then

(1) $d(\{\lambda(1), \ldots, \lambda(l)\})=k+1$ if $l \geq n+1$;

(2) $d(\{\lambda(1), \ldots, \lambda(l)\}) \geq l-(n-k)$ if $l \leq n+1$.

Denote $\mathbb{R}(a, b]=\{x \mid x$ is a real number, $a<x \leq b\}$.

Theorem 0.3 (Nochka weight function; see Chen [2, 3], Nochka [4, 5]). Suppose $\left\{a_{j}\right\}_{j \in Q}$ are in $n$-subgeneral position. Then there exists a Nochka weight function $\omega: Q \rightarrow \mathbb{R}(0,1]$ and a Nochka constant $\theta \geq 1$ such that

(1) $0<\omega(j) \cdot \theta \leq 1$ for all $j \in Q$;

(2) $q-2 n+k-1=\theta \cdot\left(\sum_{j=1}^{q} \omega(j)-k-1\right)$;

(3) $(n+1) /(k+1) \leq \theta \leq(2 n-k+1) /(k+1)$;

(4) If $P \subseteq Q$ and $0<\# P \leq n+1$, then $\sum_{j \in P} \omega(j) \leq d(P)$.

Now we list some general assumptions (undefined concepts are referred to Stoll [6] or part A of Stoll [7]):

(A1) $M$ is a connected complex manifold of dimension $m$;

(A2) $\tau$ is a parabolic exhaustion on $M$;

(A3) $V$ is a Hermitian vector space with dimension $k+1 \geq 2$;

(A4) $f: M \rightarrow \mathbb{P}(V)$ is a meromorphic map;

(A5) There is a holomorphic differential form $B$ of bidegree $(m-1,0)$ on $M$;

(A6) $f$ is general for $B$;

(A7) $\tau$ majorizes $B$ and $Y$ is the majorant;

(A8) Let $\left\{a_{j}\right\}_{j \in Q}$ be elements of $\mathbb{P}\left(V^{*}\right)$ in $n$-subgeneral position with $Q=$ $\{1, \ldots, q\}$ and $q \geq 2 n-k+1$;

(A9) Let $\omega: Q \rightarrow \mathbb{R}(0,1]$ be a Nochka weight function for the family $\left\{a_{j}\right\}_{j \in Q}$ with Nochka constant $\theta$.

Remark. (A6) implies that $f$ is linearly nondegenerate. 
Assume (A1)-(A7) hold. If $A \subseteq M, t \in \mathbb{R}^{+}$, we define $A[t]=A \cap\{x \in M \mid$ $\left.\tau(x) \leq t^{2}\right\}, A\langle t\rangle=A \cap\left\{x \in M \mid \tau(x)=t^{2}\right\}$. Let $v=d d^{c} \tau, \omega=d d^{c} \log \tau$, $\sigma=d^{c} \log \tau \wedge \omega^{m-1}$. We know that they are all nonnegative. Let $\Delta_{\tau}=\{r \in$ $\left.\mathbb{R}^{+} \mid d \tau(x) \neq 0, \forall x \in M\langle r\rangle\right\}$. Then the set $\mathbb{R}^{+}-\Delta_{\tau}$ has measure zero. Stoll [6, p. 133] says for all $r \in \Delta_{\tau}, \zeta=\int_{M\langle r\rangle} \sigma$ is a positive constant. For $0 \leq p \leq k$, let $f_{p}$ be the $p$ th associated map of $f$, let $\Omega_{p}$ be the Fubini-Study form on $\mathbb{P}\left(\bigwedge_{p+1} V\right)$. Define

$$
A_{f_{p}}(t)=t^{2-2 m} \int_{M[t]} f_{p}^{*}\left(\Omega_{p}\right) \wedge v^{m-1} .
$$

Define the $p$ th characteristic function for $0<s<r$

$$
T_{f_{p}}(r, s)=\int_{s}^{r} A_{f_{p}}(t) \frac{d t}{t} .
$$

We know $T_{f_{k}}(r, s) \equiv 0$. Denote $T_{f_{-1}}(r, s) \equiv 0$.

Lemma 0.4 (Stoll [6, Lemma 10.5, p. 161]). Assume (A1)-(A7). Let $0 \leq p \leq n$. Then $T_{f_{p}}(r, s) \rightarrow \infty$ as $r \rightarrow \infty$; moreover,

$$
\lim _{r \rightarrow \infty} \frac{T_{f_{p}}(r, s)}{\log r}=A_{f_{p}}(\infty)>0 .
$$

Let $I_{p}$ be the indeterminancy of $f_{p}$. On $M-I_{p}$ define

$$
H_{p}=m ! \cdot\left(\frac{i}{2 \pi}\right)^{m-1} \cdot(-1)^{(m-1)(m-2) / 2} \cdot f_{p}^{*}\left(\Omega_{p}\right) \wedge B \wedge \bar{B} .
$$

We know $H_{p} \geq 0$. Define $h_{p}=H_{p} / v^{m}$. For all $r \in \Delta_{\tau}$, define $S_{p}(r)=$ $\frac{1}{2} \int_{M\langle r\rangle} \log h_{p} \sigma$. Let $\nu$ be a divisor on $M$ with $S=\operatorname{supp} \nu$. The counting function of $\nu$ is defined to be

$$
n_{\nu}(r)= \begin{cases}r^{2-2 m} \int_{S[r]} \nu v^{m-1} & \text { if } m>1 ; \\ \sum_{z \in S[r]} \nu(z) & \text { if } m=1 .\end{cases}
$$

Define $N_{\nu}(r, s)=\int_{s}^{r} n_{\nu}(t) d t / t$. Let $\mu_{f}^{a}$ be the $a$-divisor of $f$. Let $d_{p}$ be the zero divisor of $f_{p}$. When $p=k$, we obtain the Wronskian divisor $d_{k}$. Since we use reduced representation for $f_{0}=f$, we have always $d_{0} \equiv 0$. We denote $d_{-1} \equiv 0$. The divisor $l_{p}=d_{p-1}-2 d_{p}+d_{p+1} \geq 0$ is called the $p$ th stationary index. We have

Theorem 0.5 (Plücker Difference Formula; Stoll [6, Theorem 7.6, p. 150]). Assume (A1)-(A7). Then for almost all $s \in \Delta_{\tau}, r \in \Delta_{\tau}$ with $0<s<r$.

$$
N_{l_{p}}(r, s)+T_{f_{p-1}}(r, s)-2 T_{f_{p}}(r, s)+T_{f_{p+1}}(r, s)=S_{p}(r)-S_{p}(s)+\operatorname{Ric}_{\tau}(r, s) \text {, }
$$

where $\operatorname{Ric}_{\tau}(r, s)$ is the Ricci function of $M$ defined in Stoll [6, (7.40), p. 147].

Now we assume $V$ is a Hermitian vector space and $(\mathfrak{x} \mid \mathfrak{y})$ is the Hermitian product of $\mathfrak{x} \in V$ and $\mathfrak{y} \in V$. Let $\|\mathfrak{x}\|$ denote the norm of $\mathfrak{x}$. For $p \in$ 
$\mathbb{Z}[0, k]$, the Hermitian product $(\mid)$ induces a Hermitian product in $\Lambda_{p} V$. Let L: $\bigwedge_{p+1} V \times V^{*} \rightarrow \bigwedge_{p} V$ be the interior product defined in the usual way. If $\mathfrak{x} \in \bigwedge_{p+1} V-\{0\}, x=\mathbb{P}(\mathfrak{x}), \alpha \in V^{*}-\{0\}$ and $a=\mathbb{P}(\alpha)$, we define $E(x)=\{\mathfrak{y} \in V \mid \mathfrak{x} \wedge \mathfrak{y}=0\}$. It is a linear subspace of $V$. Denote $E[a]=$ $E[\alpha]=\{\mathfrak{x} \in V \mid \alpha(\mathfrak{x})=0\}$. It is a hyperplane. Define $\$ x \mathrm{~L} a \$=\|\mathfrak{x} \mathrm{L} \alpha\| /\|\mathfrak{x}\| \cdot\|\alpha\|$. Define $\widetilde{G}_{p}(V)=\left(\mathfrak{x}_{0} \wedge \cdots \wedge \mathfrak{x}_{p} \mid \mathfrak{x}_{j} \in V, j=0, \ldots, p\right\}$. Then $G_{p}(V)=\mathbb{P}\left(\widetilde{G}_{p}(V)\right)$ is a Grassmann manifold. For $a \in \mathbb{P}\left(V^{*}\right)$, define an $a$-divisor $\nu=\mu_{f_{p}}^{a}$ as in Stoll [6, (8.10), p. 152]. Define

$$
N_{f_{p}}(r, s, a)=\int_{s}^{r} n_{\nu}(t) \frac{d t}{t} .
$$

Define

$$
m_{f_{p}}(r, a)=\int_{M\langle r\rangle} \log \frac{1}{\$ f_{p} L a \$} \sigma .
$$

Then we have

Theorem 0.6 (Projective First Main Theorem) (Stoll [6, (8.21), p. 153]). Assume (A1)-(A7). If $a \in \mathbb{P}\left(V^{*}\right), 0 \leq p \leq k, s \in \Delta_{\tau}, r \in \Delta_{\tau}, 0<s<r$, then

$$
T_{f_{p}}(r, s)=N_{f_{p}}(r, s ; a)+m_{f_{p}}(r, a)-m_{f_{p}}(s, a)+T_{f_{p}\llcorner a}(r, s) .
$$

If $p=0$, then $T_{f\llcorner a}(r, s) \equiv 0$ implies the usual First Main Theorem (Stoll $[6,(6.63)$, p. 140]):

$$
T_{f}(r, s)=N_{f}(r, s ; a)+m_{f}(r, a)-m_{f}(s, a) .
$$

Stoll [6, Proposition 10.9, p. 163] implies

Theorem 0.7. Assume (A1)-(A7). For $0 \leq p \leq k$,

$\log T_{f_{p}}(r, s) \leq \log T_{f}(r, s)+\log ^{+} \log Y(r)+\log ^{+} \operatorname{Ric}_{\tau}^{+}(r, s)+2 \log ^{+} \log r$, where $\leq$ means the inequality holds beside a set of $\mathbb{R}$ with finite measure.

Assume (A1)-(A8). Take $s_{0} \in \Delta_{\tau}$. For $0 \leq p \leq k$ and $j \in Q$, we define

$$
\begin{gathered}
\Phi_{p}\left(a_{j}\right)=\$ f_{p} \mathrm{~L} a_{j} \$^{2}, \quad \Gamma_{p}=\max \left\{m_{f_{p}}\left(s_{0}, a_{j}\right) \mid j \in Q\right\}, \\
\beta(r)= \begin{cases}\frac{1}{2 q\left(T_{f_{p}}\left(r, s_{0}\right)+1+\Gamma_{p}\right)} & \text { if } r \geq s_{0}>0, \\
\beta\left(s_{0}\right) & \text { if } 0 \leq r<s_{0} .\end{cases}
\end{gathered}
$$

Observe that $0<\beta(r)<1$ for all $r>0$. Using these notations, we have Lemma 0.8 (Stoll [6, p. 167, Lemma 11.5]). For any $\varepsilon>0$,

$$
\begin{aligned}
& \int_{M\langle r\rangle} \log \left(\sum_{j \in Q} \frac{\Phi_{p+1}\left(a_{j}\right)}{\Phi_{p}\left(a_{j}\right)^{1-\beta(r)}} h_{p}\right)^{\sigma} \\
& \quad \leq \zeta(1+\varepsilon)^{2}\left(3 \log T_{f_{p}}(r, s)+\log Y(r)\right)+\varepsilon \log r .
\end{aligned}
$$




\section{Product to Sum Estimate}

Lemma 1.1. Given real numbers $E_{j} \geq 1$ and $\lambda_{j}>0, j=0,1, \ldots, n-1$, such that

(1) $E_{0} \geq E_{1} \geq \cdots \geq E_{n} \geq 1$,

(2) $\sum_{j=0}^{p} \lambda_{j} \leq p+1$, for all $p \in \mathbb{Z}[0, n-1]$.

Then

$$
\prod_{j=0}^{n} E_{j} \geq\left(E_{0}\right)^{\lambda_{0}} \cdots\left(E_{n-1}\right)^{\lambda_{n-1}}\left(E_{n}\right)^{n+1-\sum_{j=0}^{n-1} \lambda_{j}}
$$

Proof. Observe that when $p=0$, (2) implies $\lambda_{0} \leq 1$. We can prove it by induction in $n$, starting from $n=1$.

Theorem 1.2. Let $\left\{\alpha_{j}\right\}_{j \in Q}$ be in n-subgeneral position. Let $\omega: Q \rightarrow \mathbb{R}(0,1]$ be a Nochka weight function with Nochka constant $\theta$ for $\left\{\alpha_{j}\right\}_{j \in Q}$ as defined in Theorem 0.3. Take $A \subseteq Q$ with $0<\# A \leq n+1$. Let $\left\{E_{j}\right\}_{j \in Q}$ be a family of real numbers with $E_{j} \geq 1$. Then there exists $B \subseteq A$ such that $\left\{\alpha_{j}\right\}_{j \in B}$ is a base of $H(A)$ and such that

$$
\prod_{j \in A}\left(E_{j}\right)^{\omega(j)} \leq \prod_{j \in B} E_{j} .
$$

Remark. If $\# A=n+1$, then $H(A)=V^{*}$ and $\left\{\alpha_{j}\right\}_{j \in B}$ is a base of $V^{*}$.

Proof. Without loss of generality, we identify $A=\mathbb{Z}[0, p]$ such that

$$
E_{0} \geq E_{1} \geq \cdots \geq E_{p} .
$$

We define $j_{0}=0, B_{0}=\left\{j_{0}\right\}, A_{0}=I_{0}=\left\{j \in A \mid \alpha_{j} \in H\left(B_{0}\right)\right\}$. Then $A_{0} \subseteq A$. If $A_{0} \subsetneq A$, we construct

$$
\begin{aligned}
& j_{1}=\min \left(A-A_{0}\right), \quad B_{1}=B_{0} \cup\left\{j_{1}\right\}=\left\{j_{0}, j_{1}\right\}, \\
I_{1}= & \left\{j \in A-A_{0} \mid \alpha_{j} \in H\left(B_{1}\right)\right\}, \quad A_{1}=A_{0} \cup I_{1}=I_{0} \cup I_{1} .
\end{aligned}
$$

$A_{1}$ is a disjoint union. Observe that $A_{1} \subseteq A$. If $A_{1} \subsetneq A$, we continue such procedure to construct $A_{2}, \ldots, A_{i}$. Say we reached $A_{i-1}$ with $A_{i-1} \subsetneq A$, we continue inductively with

$$
\begin{aligned}
& j_{i}=\min \left(A-A_{i-1}\right), \quad B_{i}=B_{i-1} \cup\left\{j_{i}\right\}=\left\{j_{0}, j_{1}, \ldots, j_{i}\right\}, \\
I_{i}= & \left\{j \in A-A_{i-1} \mid \alpha_{j} \in H\left(B_{i}\right)\right\}, \quad A_{i}=A_{i-1} \cup I_{i}=I_{0} \cup I_{1} \cup \cdots \cup I_{i} .
\end{aligned}
$$

Since we have $j_{i} \in B_{i}$, we have $\alpha_{j_{i}} \in H\left(B_{i}\right)$. Now $j_{i} \in A-A_{i-1}$ implies $j_{i} \in I_{i}$. Therefore $I_{i} \neq \varnothing$. Hence $A_{0} \subsetneq A_{i} \subsetneq \cdots \subsetneq A_{i} \subseteq A$. Because $A$ is finite, the process ends with $A_{l}=A$. Then

$$
A=A_{l}=I_{0} \cup \cdots \cup I_{l}
$$

is a disjoint union. Since $j_{i} \in I_{i}$, we have $j_{i} \neq j_{k}$ if $i \neq k$. Hence $\# B_{i}=i+1$ for all $i \in \mathbb{Z}[0, l]$. These $A_{j}$ 's have the following properties:

(1) $A_{i}=I_{0} \cup I_{1} \cup \cdots \cup I_{i}$ is a disjoint union for $i \in \mathbb{Z}[0, l]$;

(2) $j_{i}=\min I_{i}$ for all $i \in \mathbb{Z}[0, l]$; 
(3) $\left\{\alpha_{j}\right\}_{j \in B_{i}}=\left\{\alpha_{j_{\lambda}}\right\}_{\lambda \in \mathbb{Z}[0, i]}$ is a base of $H\left(A_{i}\right)$;

(4) $H\left(B_{i}\right)=H\left(A_{i}\right)$ and $d\left(A_{i}\right)=i+1$ for all $i \in \mathbb{Z}[0, l]$.

The proof of these properties is direct. In fact, (1) is already proved. Also $j_{i} \in I_{i}$ has been shown. Take $j \in I_{i}$. If $i=0$, then $j_{0}=0 \leq j$. If $i>0$, then $j \in I_{i} \subseteq A-A_{i-1}$. Hence $j \geq \min \left(A-A_{i-1}\right)=j_{i}$. Thus $j_{i}=\min I_{i}$ and (2) is proved. (3) and (4) can be proved by induction in $i$ starting from $i=0$.

Now we will complete the proof of Theorem 1.2. Define $B=B_{l}$. Since $A=A_{l}$, we see that $\left\{\alpha_{j}\right\}_{j \in B}$ is a base of $H(A)$. For $i \in \mathbb{Z}[0, l]$, define

$$
\lambda_{i}=\sum_{j \in I_{i}} \omega(j)
$$

Put $\beta_{0}=0$. For $p \in \mathbb{Z}[1, l+1]$, define $\beta_{p}=\sum_{i=0}^{p-1} \lambda_{i}$. Then

$$
\beta_{p}=\sum_{i=0}^{p-1} \lambda_{i}=\sum_{i=0}^{p-1} \sum_{j \in I_{i}} \omega(j)=\sum_{j \in A_{p-1}} \omega(j) \leq d\left(A_{p-1}\right)=p,
$$

where the inequality is due to Theorem $0.3(4)$. Here

$$
\lambda_{l}=\beta_{l+1}-\beta_{l} \leq l+1-\beta_{l} .
$$

Now Lemma 1.1 implies

$$
\prod_{j \in B} E_{j}=\prod_{i=0}^{l} E_{j_{i}} \geq\left(E_{j_{0}}\right)^{\lambda_{0}} \cdots\left(E_{j_{l-1}}\right)^{\lambda_{l-1}}\left(E_{j_{l}}\right)^{l+1-\beta_{l}} \geq \prod_{i=0}^{l}\left(E_{j_{i}}\right)^{\lambda_{i}} .
$$

Since $j_{i}=\min I_{i},(1.2)$ implies that $E_{j} \leq E_{j_{i}}$ for all $j \in I_{i}$. Hence

$$
\begin{aligned}
\prod_{j \in B} E_{j} & \stackrel{(1.5)}{\geq} \prod_{i=0}^{l}\left(E_{j_{i}}\right)^{\lambda_{i}} \stackrel{(1.4)}{=} \prod_{i=0}^{l} \prod_{j \in I_{i}}\left(E_{j_{i}}\right)^{\omega(j)} \\
& \geq \prod_{i=0}^{l} \prod_{j \in I_{i}}\left(E_{j}\right)^{\omega(j)} \stackrel{(1.3)}{=} \prod_{j \in A}\left(E_{j}\right)^{\omega(j)} \cdot \text { Q.E.D. }
\end{aligned}
$$

For $x \in G_{p}(V), d \geq 0$ and $P \subseteq Q$, define

$$
I_{x}(d, P)=\left\{j \in P \mid \$ x\left\llcorner a_{j} \$^{2} \leq d\right\} .\right.
$$

Abbreviate

$$
I_{x}=I_{x}(0, Q)=\left\{j \in Q \mid \$ x\left\llcorner a_{j} \$^{2}=0\right\} .\right.
$$

Then we have the following result.

Lemma 1.3. Suppose $\left\{a_{j} \in \mathbb{P}\left(V^{*}\right) \mid j \in Q\right\}$ are in $n$-subgeneral position. Then there is a constant $c \in \mathbb{R}(0,1)$ such that

$$
\# I_{x}(c, Q) \leq n-p \quad \text { for all } x \in G_{p}(V) \text {. }
$$


Proof. Take any $x \in G_{p}(V)$. Vectors $\mathfrak{x}_{0}, \ldots, \mathfrak{x}_{p}$ exist in $V$ such that $\mathfrak{x}=$ $\mathfrak{x}_{0} \wedge \cdots \wedge \mathfrak{x}_{p} \neq 0$ and $\mathbb{P}(\mathfrak{x})=x$. A linear subspace $E=\bigcap_{j \in I_{x}} E\left[a_{j}\right]$ is defined. If $j \in I_{x}$, then $\$ x\left\llcorner a_{j} \$=0\right.$ implies

$$
0=\mathfrak{x} L \alpha_{j}=\sum_{\lambda=0}^{p}(-1)^{\lambda} \alpha_{j}\left(\mathfrak{x}_{\lambda}\right) \mathfrak{x}_{0} \wedge \cdots \wedge \mathfrak{x}_{\lambda-1} \wedge \mathfrak{x}_{\lambda+1} \wedge \cdots \wedge \mathfrak{x}_{p} .
$$

Since the vectors $\mathfrak{x}_{0}, \ldots, \mathfrak{x}_{p}$ are linearly independent, therefore $\left\{\mathfrak{x}_{0} \wedge \cdots \wedge\right.$ $\left.\mathfrak{x}_{\lambda-1} \wedge \mathfrak{x}_{\lambda+1} \wedge \cdots \wedge \mathfrak{x}_{p} \mid \lambda=0, \ldots, p\right\}$ are linearly independent and hence we have $\alpha_{j}\left(\mathfrak{x}_{\lambda}\right)=0$ for $\lambda=0, \ldots, p$. Since $j \in I_{x}$ is chosen arbitrarily, therefore $\mathfrak{x}_{\lambda} \in E\left[a_{j}\right]$ for $j \in I_{x}$. Hence $\mathfrak{x}_{\lambda} \in E$ for $\lambda=0, \ldots, p$. Because $\left\{\mathfrak{x}_{0}, \ldots, \mathfrak{x}_{p}\right\}$ is a base of $E(x)$, we obtain $E(x) \subseteq E$.

Let $H\left(I_{x}\right)$ be the vector space generated by $\left\{\alpha_{j}\right\}_{j \in I_{x}}$. There is a subset $B \subseteq I_{x}$ such that $\left\{\alpha_{j}\right\}_{j \in B}$ is a base of $H\left(I_{x}\right)$. Define $\widetilde{E}=\bigcap_{j \in B} E\left[a_{j}\right], l=\# I_{x}$, $s=\# B$. Then $k+1-s=\operatorname{dim} \widetilde{E}$. Also $\widetilde{E} \supseteq E$ since $B \subseteq I_{x}$. If $\mathfrak{y} \in \widetilde{E}$, then $\alpha_{j}(\mathfrak{y})=0$ for all $j \in B$. Hence $\alpha(\mathfrak{y})=0$ for all $\alpha \in H\left(I_{x}\right)$. Hence $\alpha_{j}(\mathfrak{y})=0$ for all $j \in I_{x}$. Hence $\mathfrak{y} \in E$. We see that $\widetilde{E}=E \supseteq E(x)$. Therefore $p+1 \leq k+1-s$, i.e., $s \leq k-p$.

If $l=\# I_{x} \geq n+1$, then $\left\{\alpha_{j}\right\}_{j \in I_{x}}$ generates $V^{*}$. Therefore $s=k+1$ and $0<p+1 \leq k+1-(k+1)=0$, which is impossible. Hence $l<n+1$. Lemma 0.2 implies $s=\# B=\operatorname{dim} H\left(I_{x}\right) \geq l-(n-k)$. Therefore $l-(n-k) \leq s \leq k-p$, we obtain

$$
\# I_{x}=l \leq n-p .
$$

Pick $c_{x} \in \mathbb{R}(0,1)$ such that $\$ x \mathrm{~L} a_{j} \$^{2}>c_{x}$ for all $j \in Q-I_{x}$. Since $\$ y \mathrm{~L} a_{j} \$$ is continuous in $y$ near $x$, therefore there exists an open neighborhood $U_{x}$ of $x$ in $G_{p}(V)$ such that $\$ y L a_{j} \$^{2}>c_{x}$ for all $y \in U_{x}$ and $j \in Q-I_{x}$. Finitely many points $x_{1}, \ldots, x_{t}$ exist such that $G_{p}(V)=U_{x_{1}} \cup \cdots \cup U_{x_{t}}$. Define

$$
c=\min \left(c_{x_{1}}, \ldots, c_{x_{t}}\right) \in \mathbb{R}(0,1) .
$$

Take any $y \in G_{p}(V)$. Then $\lambda \in \mathbb{Z}[1, t]$ exists such that $y \in U_{x_{\lambda}}$. Take $j \in I_{y}(c)$. Assume that $j \notin I_{x_{\lambda}}$. Then $\$ y L a_{j} \$^{2}>c_{x_{\lambda}} \geq c \geq \$ y L a_{j} \$^{2}$, which is impossible. Hence $I_{y}(c, Q) \subseteq I_{x_{\lambda}}$, \#I $I_{y}(c, Q) \leq \# I_{x_{\lambda}}$. Applying (1.9) we obtain $\# I_{x_{\lambda}} \leq n-p$. Therefore $\# I_{y}(c, Q) \leq n-p$ for all $y \in G_{p}(V)$. Q.E.D.

Let $\mathscr{L}$ be the set of all subsets $B$ of $Q$ such that $\left\{\alpha_{j}\right\}_{j \in B}$ is a base of $V^{*}$. Then $\# B=k+1$ for all $B \in \mathscr{L}$. We have

Lemma 1.4. Suppose $x \in G_{p}(V)$ and $a_{j} \in \mathbb{P}\left(V^{*}\right), j \in Q$, are in n-subgeneral position. Then there is a constant $\hat{c} \in \mathbb{R}(0,1)$ such that

$$
\# I_{x}(\hat{c}, B) \leq k-p \text { for all } x \in G_{p}(V) \text { and all } B \in \mathscr{L} \text {. }
$$

Proof. $\{\alpha\}_{j \in B}$ is in general position, i.e., in $k$-subgeneral position. By Lemma 1.3 , there is a constant $c(B) \in \mathbb{R}(0,1)$ such that $\# I_{x}(c(B), B) \leq k-p$ for 
all $x \in G_{p}(V)$. Because $\mathscr{L}$ is finite, we may let $\widehat{c}=\min \{c(B) \mid B \in \mathscr{L}\} \in$ $\mathbb{R}(0,1)$. Then $I_{x}(\hat{c}, B) \subseteq I_{x}(c(B), B)$ for all $B \in \mathscr{L}$ and all $x \in G_{p}(V)$. Hence $\# I_{x}(\hat{c}, B) \leq k-p$ for all $x \in G_{p}(V)$ and $B \in \mathscr{L}$. Q.E.D.

Take $p \in \mathbb{Z}[0, k-1]$. Lemma 1.3 shows there is a constant $c \in \mathbb{R}(0,1)$ such that $\# I_{x}(c, Q) \leq n-p$ for all $x \in G_{p}(V)$. Lemma 1.4 shows there is a constant $\hat{c} \in \mathbb{R}(0,1)$ such that $\# I_{x}(\hat{c}, B) \leq k-p$ for all $x \in G_{p}(V)$ and $B \in \mathscr{L}$. Fix these constants and define

$$
c_{p}=c^{-q} \hat{c}^{-(p+1)}(k-p)^{-(k-p)}>0 .
$$

Theorem 1.5 (Product to Sum Estimate). Assume (A3) and (A8), take $p \in$ $\mathbb{Z}[0, k-1]$, and take $x \in G_{p}(V)$ and $y \in G_{p+1}(V)$ with $E(x) \subsetneq E(y)$. Assume that $E(x) \nsubseteq E\left[a_{j}\right]$ for all $j \in Q$. Take $\beta_{j} \in \mathbb{R}[0,1]$ for each $j \in Q$. Then we have the estimate

$$
\prod_{j=1}^{q} \frac{\$ y \mathrm{~L} a_{j} \$^{2 \omega(j)}}{\$ x \mathrm{~L} a_{j} \$^{2 \omega(j)-2 \beta_{j}}} \leq c_{p}\left(\sum_{j=1}^{q} \frac{\$ y \mathrm{~L} a_{j} \$^{2}}{\$ x \mathrm{~L} a_{j} \$^{2-2 \beta_{j}}}\right)^{k-p} .
$$

Proof. Since $E(x) \nsubseteq E\left[a_{j}\right]$ we have $\$ x\left\llcorner a_{j} \$>0\right.$. Define

$$
F_{j}=\frac{\$ y\left\llcorner a_{j} \$^{2}\right.}{\$ x \mathrm{~L} a_{j} \$^{2}} \in \mathbb{R}, \quad G_{j}=\$ x \mathrm{~L} a_{j} \$^{2 \beta_{j}} \leq 1 .
$$

Then $F_{j} \geq 1$. We have also $0<G_{j}=\$ x \mathrm{~L} a_{j} \$^{2 \beta_{j}} \leq 1$. Take $c$ and $\hat{c}$ as in (1.10). Then $\# I_{x}(c, Q) \leq n-p$. A subset $A$ of $Q$ exists such that $\# I_{x}(c) \subsetneq A$ and $\# A=n+1$. Then $\left\{\alpha_{j}\right\}_{j \in A}$ generates $V^{*}$. By Theorem 1.2, there is a subset $B \subseteq A$ such that $\left\{\alpha_{j}\right\}_{j \in B}$ is a base of $V^{*}$ and such that

$$
\prod_{j \in A}\left(F_{j}\right)^{\omega(j)} \leq \prod_{j \in B} F_{j} .
$$

Observe that $B \in \mathscr{L}$. If $j \in Q-A$, then $j \notin I_{x}(c, Q)$. Hence $\$ x \operatorname{L} a_{j} \$^{2} \geq c$. Thus $F_{j} \leq 1 / c$ and $\sum_{j \in Q-A} \omega(j) \leq \#(Q-A) \leq q$. Since $c<1$, we have

$$
\prod_{j \in Q-A}\left(F_{j}\right)^{\omega(j)} \leq c^{-q} .
$$

Therefore

$$
\prod_{j \in Q}\left(F_{j}\right)^{\omega(j)} \leq c^{-q} \prod_{j \in A}\left(F_{j}\right)^{\omega(j)} \leq c^{-q} \prod_{j \in B} F_{j} .
$$

Because $B \in \mathscr{L}$, we have $\# I_{x}(\hat{c}, B) \leq k-p$. Hence a subset $I$ of $B$ exists such that $\# I=k-p$ and $I_{x}(\hat{c}, B) \subseteq I$. Hence $\#(B-I)=k+1-(k-p)=p+1$ and

$$
\prod_{j \in B-I} F_{j} \leq \hat{c}^{-(p+1)} .
$$


We obtain

$$
\prod_{j \in Q}\left(F_{j}\right)^{\omega(j)} \leq c^{-q} \prod_{j \in B} F_{j} \leq c^{-q} \hat{c}^{-(p+1)} \prod_{j \in I} F_{j}
$$

Hence

$$
\begin{aligned}
\prod_{j \in Q}\left[\left(F_{j}\right)^{\omega(j)} G_{j}\right] & =\prod_{j \in Q}\left(F_{j}\right)^{\omega(j)} \cdot \prod_{j \in Q} G_{j} \\
& \leq c^{-q} \hat{c}^{p+1}\left(\prod_{j \in I} F_{j}\right)\left(\prod_{j \in I} G_{j}\right) \\
& =c^{-q} \hat{c}^{-(p+1)} \prod_{j \in I}\left(F_{j} G_{j}\right) .
\end{aligned}
$$

On the other hand, we have

$$
\left(\prod_{j \in I} F_{j} G_{j}\right)^{1 /(k-p)} \leq \frac{1}{k-p}\left(\sum_{j \in I} F_{j} G_{j}\right) \leq \frac{1}{k-p}\left(\sum_{j \in Q} F_{j} G_{j}\right),
$$

since the arithmetic mean majorizes the geometric mean. Therefore

$$
\begin{aligned}
& \prod_{j \in Q} \frac{\$ y L a_{j} \$^{2 \omega(j)}}{\$ x \mathrm{~L} a_{j} \$^{2 \omega(j)-2 \beta_{j}}}=\prod_{j \in Q}\left[\left(F_{j}\right)^{\omega(j)} G_{j}\right] \\
& \leq c^{-q} \hat{c}^{-(p+1)}(k-p)^{-(k-p)}\left(\sum_{j \in Q} F_{j} G_{j}\right)^{k-p} \\
& \quad=c_{p}\left(\sum_{j \in Q} \frac{\$ y \mathrm{~L} a_{j} \$^{2}}{\$ x \mathrm{~L} a_{j} \$^{2-2 \beta_{j}}}\right)^{k-p}
\end{aligned}
$$

and (1.11) is true. Q.E.D.

2. DEFECT RELATIONS FOR HYPERPLANES IN SUBGENERAL POSITION Assume (A1)-(A9). For $p \in \mathbb{Z}[0, k]$ and $j \in Q$, take $s_{0} \in \Delta_{\tau}$, define

$$
F_{j p}=\frac{\Phi_{p+1}\left(a_{j}\right)}{\Phi_{p}\left(a_{j}\right)}
$$

take $c_{p}$ as in (1.10), and define

$$
\lambda_{p}=1+\zeta \log c_{p} .
$$

Lemma 2.1. Assume that (A1)-(A9) hold. Take $p \in \mathbb{Z}[0, k-1]$. Define $\beta(r)$ as in $§ 1$. Then we have

$$
\begin{array}{r}
2(k-p) S_{p}(r)+2 \sum_{j \in Q} \omega(j)\left(m_{f_{p}}\left(r, a_{j}\right)-m_{f_{p+1}}\left(r, a_{j}\right)\right) \\
\leq \lambda_{p}+(k-p) \int_{M\langle r\rangle} \log \left(\sum_{j \in Q} F_{j p} \Phi_{p}\left(a_{j}\right)^{\beta(r)} h_{p}\right) \sigma
\end{array}
$$

for almost all $r>1$. 
Proof. The Product to Sum Estimate (Theorem 1.5) reads

$$
\prod_{j \in Q}\left(F_{j p}\right)^{\omega(j)} \Phi_{p}\left(a_{j}\right)^{\beta(r)} \leq c_{p}\left(\sum_{j \in Q} F_{j p} \Phi_{p}\left(a_{j}\right)^{\beta(r)}\right)^{k-p} .
$$

If $r>s_{0}$, we obtain

$$
\begin{aligned}
\lambda_{p}+ & (k-p) \int_{M\langle r\rangle} \log \left(\sum_{j \in Q} F_{j p} \Phi_{p}\left(a_{j}\right)^{\beta(r)} h_{p}\right) \sigma \\
= & 1+\int_{M\langle r\rangle} \log c_{p}\left(\sum_{j \in Q} F_{j p} \Phi_{p}\left(a_{j}\right)^{\beta(r)}\right)^{k-p} \sigma+2(k-p) S_{f_{p}}(r) \\
\geq & 1+\int_{M\langle r\rangle} \log \left(\prod_{j \in Q}\left(F_{j p}\right)^{\omega(j)} \Phi_{p}\left(a_{j}\right)^{\beta(r)}\right) \sigma+2(k-p) S_{f_{p}}(r) \\
= & 1+\sum_{j \in Q} \beta(r) \int_{M\langle r\rangle} \log \Phi_{p}\left(a_{j}\right) \sigma+\sum_{j \in Q} \omega(j) \int_{M\langle r\rangle} \log F_{j p} \sigma+2(k-p) S_{f_{p}}(r) \\
= & 1-2 \beta(r) \sum_{j \in Q} m_{f_{p}}\left(r, a_{j}\right) \\
& +2 \sum_{j \in Q} \omega(j)\left(m_{f_{p}}\left(r, a_{j}\right)-m_{f_{p+1}}\left(r, a_{j}\right)\right)+2(k-p) S_{f_{p}}(r) .
\end{aligned}
$$

Theorem 0.6 with $s=s_{0}$ implies that $m_{f_{p}}\left(r, a_{j}\right) \leq T_{f_{p}}(r, s)+m_{f_{p}}\left(s_{0}, a_{j}\right)$ for almost all $r>s_{0}$. Therefore for almost all $r>s_{0}$ we have

$$
\begin{aligned}
2 \beta(r) \sum_{j \in Q} m_{f_{p}}\left(r, a_{j}\right) & \leq 2 \beta(r)\left(q T_{f_{p}}\left(r, s_{0}\right)+\sum_{j \in Q} m_{f_{p}}\left(s_{0}, a_{j}\right)\right) \\
& \leq 2 \beta(r) q\left(T_{f_{p}}\left(r, s_{0}\right)+\Gamma_{p}\right) \\
& \leq 2 \beta(r) q\left(T_{f_{p}}\left(r, s_{0}\right)+\Gamma_{p}+1\right)=1 .
\end{aligned}
$$

Thus we obtain the desired estimate (2.2). Q.E.D.

Lemma 2.2. Assume (A1)-(A9). For any $\varepsilon>0$,

$$
\begin{aligned}
& \sum_{j \in Q} \omega(j)\left(m_{f_{p}}\left(r, a_{j}\right)-m_{f_{p+1}}\left(r, a_{j}\right)\right)+(k-p) S_{p}(r) \leq \frac{1}{2}(k-p) \zeta(1+\varepsilon)^{2} \\
& \quad \times\left(3 \log T_{f}(r, s)+\log Y(r)+3 \log ^{+} \log Y(r)+3 \log ^{+} \operatorname{Ric}_{\tau}^{+}(r, s)\right)+\varepsilon \log r . \\
& \text { Proof. Abbreviate } S=(k-p) S_{p}(r)+\sum_{j \in Q} \omega(j)\left(m_{f_{p}}\left(r, a_{j}\right)-m_{f_{p+1}}\left(r, a_{j}\right)\right) .
\end{aligned}
$$


Then Lemma 2.1 and Lemma 0.8 give us

$$
\begin{aligned}
S & \leq \frac{1}{2} \lambda_{p}+\frac{1}{2}(k-p) \int_{M\langle r\rangle} \log \left(\sum_{j \in Q} F_{j} \Phi_{p}\left(a_{j}\right)^{\beta(r)} h_{p}\right) \sigma \\
& \leq \frac{1}{2} \lambda_{p}+\frac{1}{2}(k-p)\left(\zeta(1+\varepsilon)^{2}\left(3 \log T_{f_{p}}(r, s)+\log Y(r)\right)+\varepsilon \log r\right) .
\end{aligned}
$$

Theorem 0.7 implies

$$
\log T_{f_{p}}(r, s) \leq \log T_{f}(r, s)+\log ^{+} \log Y(r)+\log ^{+} \operatorname{Ric}_{\tau}^{+}(r, s)+\frac{\varepsilon}{\zeta(1+\varepsilon)^{2}} \log r .
$$

Hence

$$
\begin{aligned}
S \leq \frac{1}{2}(k-p) \zeta(1+\varepsilon)^{2}( & 3 \log T_{f}(r, s)+\log Y(r) \\
& \left.+3 \log ^{+} \log Y(r)+3 \log ^{+} \operatorname{Ric}_{\tau}^{+}(r, s)\right)+3(k-p) \varepsilon \log r .
\end{aligned}
$$

If we start with $\varepsilon / 3(k-p)$ instead of $\varepsilon$, then we get the lemma. Q.E.D.

Theorem 2.3 (Second Main Theorem). Assume (A1)-(A9). Take any $\varepsilon>0$. Then

$$
\begin{aligned}
N_{d_{k}}(r, s) & +\sum_{j \in Q} \omega(j) m_{f}\left(r, a_{j}\right) \\
\leq & (k+1) T_{f}(r, s)+\frac{k(k+1)}{2} \operatorname{Ric}_{\tau}(r, s)+\varepsilon \log r+\frac{1}{4} k(k+1) \zeta(1+\varepsilon)^{2} \\
& \times\left(3 \log T_{f}(r, s)+\log Y(r)+3 \log ^{+} \log Y(r)+3 \log ^{+} \operatorname{Ric}_{\tau}^{+}(r, s)\right) .
\end{aligned}
$$

Proof. Since $m_{f_{k}}\left(r, a_{j}\right)=0$, we have

$$
\sum_{p=0}^{k-1} \sum_{j \in Q}\left(m_{f_{p}}\left(r, a_{j}\right)-m_{f_{p+1}}\left(r, a_{j}\right)\right)=\sum_{j \in Q} m_{f}\left(r, a_{j}\right) .
$$

Summing up (2.3) over $p=0, \ldots, k-1$ gives

$$
\begin{aligned}
\sum_{j \in Q} \omega(j) m_{f}\left(r, a_{j}\right)+\sum_{p=0}^{k-1}(k-p) S_{p}(r) \leq \frac{1}{4} k(k+1) \zeta(1+\varepsilon)^{2} \\
\times\left(3 \log T_{f}(r, s)+\log Y(r)+\log ^{+} \log Y(r)+3 \log ^{+} \operatorname{Ric}_{\tau}^{+}(r, s)\right)+k \varepsilon \log r .
\end{aligned}
$$

Theorem 0.5 (Plücker Difference Formula) implies

$$
\begin{gathered}
\sum_{p=0}^{k-1}(k-p)\left(N_{l_{p}}(r, s)+T_{f_{p+1}}(r, s)-2 T_{f_{p}}(r, s)+T_{f_{p-1}}(r, s)\right) \\
=\sum_{p=0}^{k-1}(k-p)\left(S_{p}(r)-S_{p}(s)\right)+\frac{k(k+1)}{2} \operatorname{Ric}_{\tau}(r, s),
\end{gathered}
$$


where

$$
N_{l_{p}}(r, s)=N_{d_{p+1}}(r, s)-2 N_{d_{p}}(r, s)+N_{d_{p-1}}(r, s) .
$$

Since $T_{-1}(r, s)=0=T_{k}(r, s)$ and $d_{0}=d_{-1}=0,(2.6)$ and (2.7) imply

$$
N_{d_{k}}(r, s)-(k+1) T_{f}(r, s)=\sum_{p=0}^{k-1}(k-p)\left(S_{p}(r)-S_{p}(s)\right)+\frac{k(k+1)}{2} \operatorname{Ric}_{\tau}(r, s) \text {. }
$$

Combining this with (2.5) gives

$$
\begin{aligned}
N_{d_{k}}(r, s)-(k+1) T_{f}(r, s) & \\
\leq & -\sum_{j \in Q} \omega(j) m_{f}\left(r, a_{j}\right)+k \varepsilon \log r+\frac{k(k+1)}{2} \operatorname{Ric}_{\tau}(r, s) \\
& +\frac{1}{4} k(k+1) \zeta(1+\varepsilon)^{2}\left(3 \log T_{f}(r, s)+\log Y(r)\right. \\
& \left.+3 \log { }^{+} \log Y(r)+3 \log ^{+} \operatorname{Ric}_{\tau}^{+}(r, s)\right) .
\end{aligned}
$$

Therefore if we start with $\varepsilon / k$ instead of $\varepsilon$, we obtain the theorem. Q.E.D.

Definition 2.4. Assume (A1)-(A9). Define

$$
\begin{array}{ll}
\text { the Nevanlinna defect } & \delta_{f}(a)=\varliminf_{r \rightarrow \infty} \frac{m_{f}(r, a)}{T_{f}(r, s)} ; \\
\text { the Ricci defect } & R_{f}=\varlimsup_{r \rightarrow \infty} \frac{\operatorname{Ric}_{\tau}^{+}(r, s)}{T_{f}(r, s)} ; \\
\text { the majorant defect } & Y_{f}=\varlimsup_{r \rightarrow \infty} \frac{\log Y(r)}{T_{f}(r, s)} ; \\
\text { the ramification defect } & D_{f}=\varliminf_{r \rightarrow \infty} \frac{N_{d_{k}}(r, s)}{T_{f}(r, s)} .
\end{array}
$$

Then by First Main Theorem (Theorem 0.6), $0 \leq \delta_{f}(a) \leq 1$.

Recall that $\zeta=\int_{M\langle r\rangle} \sigma>0$ is a constant for all $r \in \Delta_{\tau}$.

Theorem 2.5 (Defect Relations). Assume (A1)-(A9). Then

$$
\frac{n+1}{k+1} D_{f}+\sum_{j \in Q} \delta_{f}\left(a_{j}\right) \leq(2 n-k+1)+\frac{k(2 n-k+1)}{2}\left(R_{f}+\frac{\zeta}{2} Y_{f}\right) .
$$

Moreover, the constant term $2 n-k+1$ on the right-hand side is a sharp bound. Proof. Theorem 0.4 implies

$$
\lim _{r \rightarrow \infty} \frac{\log r}{T_{f}(r, s)}<\infty .
$$

Dividing both sides of (2.4) in Theorem 2.3 by $T_{f}(r, s)$ and letting $r \rightarrow \infty$ and $\varepsilon \rightarrow 0$, we obtain

$$
D_{f}+\sum_{j \in Q} \omega(j) \delta_{f}\left(a_{j}\right) \leq k+1+\frac{k(k+1)}{2} R_{f}+\frac{k(k+1) \zeta}{4} Y_{f} .
$$


Multiplying both sides of (2.9) by the Nochka constant $\theta \geq 1$, we obtain $\theta D_{f}+\sum_{j \in Q} \delta_{f}\left(a_{j}\right) \leq \sum_{j \in Q}(1-\theta \omega(j)) \delta_{f}\left(a_{j}\right)+\theta(k+1)+\frac{\theta k(k+1)}{2}\left(R_{f}+\frac{\zeta}{2} Y_{f}\right)$.

Notice that $0 \leq \delta_{f}\left(a_{j}\right) \leq 1$ and Theorem 0.3 implies $1-\theta \omega(j) \geq 0$ and $q-\theta\left(\sum_{j \in Q} \omega(j)-k-1\right)=2 n-k+1$. Hence we have

$$
\theta D_{f}+\sum_{j \in Q} \delta_{f}\left(a_{j}\right) \leq(2 n-k+1)+\frac{\theta k(k+1)}{2}\left(R_{f}+\frac{\zeta}{2} Y_{f}\right)
$$

Since

$$
\frac{n+1}{k+1} \leq \theta \leq \frac{2 n-k+1}{k+1}
$$

(Theorem 0.3), we have

$$
\frac{n+1}{k+1} D_{f}+\sum_{j \in Q} \delta_{f}\left(a_{j}\right) \leq(2 n-k+1)+\frac{k(2 n-k+1)}{2}\left(R_{f}+\frac{\zeta}{2} Y_{f}\right) .
$$

To derive the last inequality from (2.10) we have to define $R_{f} \geq 0$.

The sharpness comes from the example in Nochka [4]. Q.E.D.

When $M=\mathbb{C}^{m}$, we may use the usual metric and the usual differential operator on it; then $\operatorname{Ric}_{\tau}(r, s)=0$ and $Y(r)=1$. Therefore we have the following corollary. The statement (1) coincides with the result of Stoll [6, Theorem 12.1, p. 175].

Corollary 2.6. Assume (A1)-(A9).

(1) If $k=n$, namely, if $\left\{a_{j}\right\}_{j \in Q}$ are in general position, then

$$
D_{f}+\sum_{j \in Q} \delta_{f}\left(a_{j}\right) \leq k+1+\frac{k(k+1)}{2}\left(R_{f}+\frac{\zeta}{2} Y_{f}\right)
$$

(2) if $M=\mathbb{C}^{m}$, then

$$
\frac{n+1}{k+1} D_{f}+\sum_{j \in Q} \delta_{f}\left(a_{j}\right) \leq 2 n-k+1
$$

\section{DEFECT RELATIONS FOR $k$-DEGENERATE MEROMORPHIC MAPS}

Now we shall deal with the case where $f$ is linearly degenerate while $\left\{a_{j}\right\}_{j \in Q}$ are in general position.

Let $W$ be a Hermitian vector space of dimension $n+1$. Let $V$ be a linear subspace of dimension $k+1$ with $1 \leq k \leq n$. The Hermitian metric on $W$ restricts to a Hermitian metric on $V$. The inclusion map $\rho: V \rightarrow W$ is an isometry. It induces an injective, projective linear map $\hat{\rho}: \mathbb{P}(V) \rightarrow \mathbb{P}(W)$ such that

$$
\hat{\rho} \circ \mathbb{P}=\mathbb{P} \circ \rho
$$


Also $\rho$ induces a surjective linear map $\rho^{*}: W^{*} \rightarrow V^{*}$, the dual linear map, which is well defined by the identity

$$
\rho^{*}(\alpha)(\mathfrak{x})=\alpha(\rho(\mathfrak{x}))
$$

for all $\mathfrak{x} \in V$ and $\alpha \in W^{*}$. The Hermitian metrics on $W$ and $V$ induce metrics on $W^{*}$ and $V^{*}$. If $\alpha \in W^{*}$, then

$$
\rho^{*}(\alpha)=\alpha \circ \rho=\left.\alpha\right|_{V} .
$$

The kernel

$$
K=\operatorname{ker} \rho^{*}
$$

is a linear subspace of dimension $n-k$ in $W^{*}$. Let $L=K^{\perp}$ be the orthonormal complementary subspace of $K$ in $W^{*}$. Then $W^{*}=K \oplus L$ and $\operatorname{dim} L=k+1$. We have exact sequences:

$$
\begin{aligned}
& 0 \rightarrow V \stackrel{\rho}{\stackrel{\rho}{\rightarrow} W} \rightarrow V^{\perp} \rightarrow 0, \\
& 0 \leftarrow V^{*} \stackrel{\rho^{*}}{\leftarrow} W^{*} \leftarrow K \leftarrow 0, \\
& \quad \eta \uparrow \quad\|\quad\| \\
& 0 \leftarrow L \leftarrow K \oplus L \leftarrow K \leftarrow 0 .
\end{aligned}
$$

We know that $\eta=\left.\rho^{*}\right|_{L}: L \rightarrow V^{*}$ is a linear isomorphism. In fact, $\eta=$ $\left.\rho^{*}\right|_{L}: L \rightarrow V^{*}$ is an isometry and so we may identify $V^{*}$ with $L$ such that $\eta$ becomes the identity.

Lemma 3.1. Let $\Omega(W)$ be the Fubini-Study form on $\mathbb{P}(W)$. Let $\Omega(V)$ be the one on $\mathbb{P}(V)$. Then

$$
\hat{\rho}^{*}(\Omega(W))=\Omega(V),
$$

where ${ }^{*}$ indicates the pull back map between differential forms.

Given $a \in \mathbb{P}\left(W^{*}\right)$, suppose $0 \neq \alpha \in W^{*}$ and $a=\mathbb{P}(\alpha)$. Then

$$
\Delta_{\rho}(a)= \begin{cases}\frac{\left\|\rho^{*}(\alpha)\right\|}{\|\alpha\|}>0 & \text { if } V \nsubseteq E[a] ; \\ 0 & \text { if } V \subseteq E[a]\end{cases}
$$

is well defined and is independent on the choice of $\alpha$. We can write $\alpha=\beta+\gamma$ with $\beta \in K$ and $\gamma \in L$. Then $\|\alpha\|^{2}=\|\beta\|^{2}+\|\gamma\|^{2},\left\|\rho^{*}(\alpha)\right\|=\left\|\rho^{*}(\gamma)\right\|=\|\gamma\|$. Hence

$$
0 \leq \Delta_{\rho}(a)=\frac{\|\gamma\|}{\|\alpha\|} \leq 1
$$

where $\Delta_{\rho}(a)=0$ iff $\alpha=\beta \in K$.

Take $p \in \mathbb{Z}[1, k]$. The inclusion $\rho: V \rightarrow W$ induces an inclusion $\rho_{p}$ : $\bigwedge_{p+1} V \rightarrow \bigwedge_{p+1} W$ by $\rho_{p}\left(\mathfrak{x}_{0} \wedge \cdots \wedge \mathfrak{x}_{p}\right)=\rho\left(\mathfrak{x}_{0}\right) \wedge \cdots \wedge\left(\mathfrak{x}_{p}\right)$. The Hermitian metrics on $W$ and $V$ induce Hermitian metrics on $\Lambda_{p+1} V$ and $\Lambda_{p+1} W$ as usual. Then $\rho_{p}$ is an isometry. $\rho_{p}$ induces an inclusion map $\hat{\rho}_{p}: G_{p}(V) \rightarrow G_{p}(W)$. Take $x \in \mathbb{P}\left(\bigwedge_{p+1} V\right), a \in \mathbb{P}\left(W^{*}\right)-\mathbb{P}(K)$. Suppose $\alpha \in W^{*}-K$ satisfying $\mathbb{P}(\alpha)=a$. Then $\rho^{*}(\alpha) \neq 0$, so $b=\hat{\rho}^{*}(a)=\mathbb{P}\left(\rho^{*}(\alpha)\right)$ is well defined. Moreover we have the following lemma. 


\section{Lemma 3.2.}

$$
\$ \hat{\rho}_{p}(x) \mathrm{L} a \$=\$ x \mathrm{~L} b \$ \cdot \Delta_{\rho}(a) .
$$

Proof. Define $\rho_{-1}: \mathbb{C} \rightarrow \mathbb{C}$ to be the identity. Take $\mathfrak{x} \in \Lambda_{p+1} V$ such that $x=\mathbb{P}(\mathfrak{x})$. Then $\left\|\rho_{p}(\mathfrak{x})\right\|=\|\mathfrak{x}\|$ and $b=\mathbb{P} \circ \rho^{*}(\alpha)$. Therefore

$$
\begin{aligned}
\$ \hat{\rho}_{p}(x) \mathrm{L} a \$ & =\frac{\left\|\rho_{p}(\mathfrak{x}) \mathrm{L} \alpha\right\|}{\left\|\rho_{p}(\mathfrak{x})\right\| \cdot\|\alpha\|}=\frac{\left\|\rho_{p-1}\left(\mathfrak{x} \mathrm{L} \rho^{*}(\alpha)\right)\right\|}{\|\mathfrak{x}\| \cdot\|\alpha\|} \\
& =\frac{\left\|\mathfrak{x} \mathrm{L} \rho^{*}(\alpha)\right\|}{\|\mathfrak{x}\| \cdot\left\|\rho^{*}(\alpha)\right\|} \frac{\left\|\rho^{*}(\alpha)\right\|}{\|\alpha\|}=\$ x \operatorname{LP}\left(\rho^{*}(\alpha)\right) \$ \cdot \Delta_{\rho}(a) \\
& =\$ x \mathrm{~L} b \$ \cdot \Delta_{\rho}(a) . \quad \text { Q.E.D. }
\end{aligned}
$$

Now we make the following general assumptions:

(B1) $M$ is a connected complex manifold of dimension $m$;

(B2) $\tau$ is a parabolic exhaustion on $M$;

(B3) Let $h, k, n$ be integers with $0 \leq h<k \leq n$. Let $W$ be a Hermitian vector space of dimension $n+1$ and $V \subseteq W$ be a Hermitian vector space with dimension $k+1$. Suppose that the Hermitian metric on $V$ is the restriction of the one on $W$. Let $\rho: V \rightarrow W$ be the inclusion map. Assume that $\rho$ induces the inclusion map $\hat{\rho}: \mathbb{P}(V) \rightarrow \mathbb{P}(W)$ with $\hat{\rho} \circ \mathbb{P}=\mathbb{P} \circ \rho$;

(B4) $g: M \rightarrow \mathbb{P}(W)$ is a $k$-degenerate meromorphic map and $f: M \rightarrow \mathbb{P}(V)$ is a meromorphic map such that $g=\hat{\rho} \circ f: M \rightarrow \mathbb{P}(W)$;

(B5) There is a holomorphic differential form $B$ of bidegree $(m-1,0)$ on $M$;

(B6) $f$ is general for $B$;

(B7) $\tau$ majorizes $B$ and $Y$ is the majorant;

(B8) Let $Q=\mathbb{Z}[1, q]$ with $q>2 n-k+1$. Suppose $a_{j} \in \mathbb{P}\left(W^{*}\right), j \in Q$, are in general position and $V \nsubseteq E\left[a_{j}\right]$ for any $j \in Q$.

Lemma 3.3 (Chen [3]). Assume (B3), (B4) and (B8). Then $\hat{\rho}^{*}\left(a_{j}\right), j \in Q$, are points of $\mathbb{P}\left(V^{*}\right)$ in $n$-subgeneral position.

Assume (B1)-(B8) hold. Then all assumptions of Theorem 2.5 are satisfied for $f$ and $\left\{\hat{\rho}^{*}\left(a_{j}\right)\right\}_{j \in Q}$. Therefore the result, the defect relation, also holds. We need only to translate the results in terms of $g$. Applying Lemma 3.1 and Lemma 3.2, direct calculations of definitions will give

Theorem 3.4. Assume (B1)-(B8). Define $b=\hat{\rho}^{*}(a) \in \mathbb{P}\left(V^{*}\right)$. Let $0<s<r \in$ $\mathbb{R}$. Then we have

$$
\begin{gathered}
T_{f}(r, s)=T_{g}(r, s), \quad N_{f}(r, s, b)=N_{g}(r, s, a), \\
\zeta \log \frac{1}{\Delta_{\rho}(a)}+m_{f}(r, b)=m_{g}(r, a), \\
\delta_{f}(b)=\delta_{g}(a), \quad R_{f}=R_{g} \quad \text { and } \quad Y_{f}=Y_{g} .
\end{gathered}
$$

Therefore Theorem 2.5 (Defect Relations) implies the following theorem. 
Theorem 3.5 (Defect Relations). Assume (B1)-(B8). Assume $R_{g}<\infty$ and $Y_{g}<\infty$. Then

$$
\frac{n+1}{k+1} D_{g}+\sum_{j \in Q} \delta_{g}\left(a_{j}\right) \leq 2 n-k+1+\frac{k(2 n-k+1)}{2}\left(R_{g}+\frac{\zeta}{2} Y_{g}\right) .
$$

Moreover the constant term $2 n-k+1$ on the right-hand side is a sharp bound. Remark. When $\operatorname{dim} M=1$, (3.7) coincides with a result of Nochka [4]. When $k=n$, it becomes a result in Stoll [6].

Now we change the condition in (B8) from being in general position to being in $N$-subgeneral position and set assumption

$\left(\right.$ B8 $\left.^{\#}\right)$ Suppose $2 N-k+1 \leq \# Q=q<\infty$. Suppose $a_{j} \in \mathbb{P}\left(W^{*}\right), j \in Q$, are in $N$-subgeneral position and $V \nsubseteq E\left[a_{j}\right]$ for all $j \in Q$.

Lemma 3.6 (Chen [3]). Assume (B1)-(B7). Let $a_{j}$ be given in $\left(\mathrm{B} 8^{\#}\right)$. Denote $\beta_{j}=\rho^{*}\left(\alpha_{j}\right)$ and $b_{j}=\mathbb{P}\left(\beta_{j}\right)$. Then $\left\{b_{j}\right\}_{j \in Q}$ are in $N$-subgeneral position in $\mathbb{P}\left(V^{*}\right)$.

When Theorems 3.4, 3.6 and 2.5 are applied, we obtain defect relations for a $k$-degenerate meromorphic map $g$ with $\left\{a_{j}\right\}_{j \in Q}$ in $N$-subgeneral position. The defect relation has exactly the same form as seen in Theorem 2.5, although here the meromorphic map is $g: M \rightarrow \mathbb{P}(W)$ and $a_{j} \in \mathbb{P}\left(W^{*}\right)$.

Theorem 3.7 (Defect Relations). Assume that (B1)-(B7) hold. Assume (B8 $\left.{ }^{\#}\right)$ holds too. Take $p \in \mathbb{Z}[0, k-1]$. Assume $R_{g}<\infty$ and $Y_{g}<\infty$. Then

$$
\frac{N+1}{k+1} D_{g}+\sum_{j \in Q} \delta_{g}\left(a_{j}\right) \leq 2 N-k+1+\frac{k(2 N-k+1)}{2}\left(R_{g}+\frac{\zeta}{2} Y_{g}\right) .
$$

Moreover the constant term $2 N-k+1$ on the right-hand side is a sharp bound.

In a similar way we can reach the Second Main Theorem and defect relation for associated maps of $g$. But the calculations are much more complicated. Therefore we state only the simplest result of it without proof. More details are in Chen [2]. First we state an assumption:

(C8) Let $\left\{a_{j}\right\}_{j \in Q}$ be elements of $G_{h}\left(W^{*}\right)$ that are in general position in $\mathbb{P}\left(\bigwedge_{h+1} W\right)$. Take $\alpha_{j} \in \bigwedge_{h+1} W^{*}$ for $j \in Q$ such that $a_{j}=\mathbb{P}\left(\alpha_{j}\right)$ and suppose that $\left.\alpha_{j}\right|_{\rho_{h}}\left(\bigwedge_{h+1} V\right) \not \equiv 0$. Suppose

$$
2\left(\begin{array}{l}
n+1 \\
h+1
\end{array}\right)-\left(\begin{array}{l}
k+1 \\
h+1
\end{array}\right) \leq \# Q=q<\infty .
$$

Theorem 3.8 (Defect Relations for Associated Maps on $\mathbb{C}^{m}$ ). Assume that $M=$ $\mathbb{C}^{m}$ and that (B3), (B4) and (B6) hold. Let $h$ and $p$ be integers satisfying $0 \leq h \leq p \leq k-1$. Assume (C8) holds too. For $r>s>0$, define the defect of $g_{p}$ at $a_{j}$ by

$$
\delta_{g_{p}}\left(a_{j}\right)=\lim _{r \rightarrow \infty} \frac{m_{g_{p}}\left(a_{j}\right)}{T_{g_{p}}(r, s)} .
$$


Then we have

$$
\sum_{j \in Q} \delta_{g_{p}}\left(a_{j}\right) \leq\left(\begin{array}{l}
n+1 \\
h+1
\end{array}\right)\left(1+\frac{h+1}{p+1}\right)-\left(\begin{array}{l}
k+1 \\
h+1
\end{array}\right) .
$$

Thanks to Professor W. Stoll for the help on this work.

\section{REFERENCES}

1. H. Cartan, Sur les zéros des combinaisons linéaires de p fonctions holomorphes donnés, Mathematica (Cluj) 7 (1933), 80-103.

2. W. Chen, Cartan's conjecture: defect relations for meromorphic maps from parabolic manifold to projective space, Ph.D. dissertation, Notre Dame University, 1987.

3. _ On subgeneral position, Notre Dame Math. Preprints, \#100, 1987.

4. E. I. Nochka, Defect relations of meromorphic curves, Izv. Akad. Nauk Moldav. SSR Ser. Fiz.-Tekn. Mat. Nauk 1982, no. 1, 41-47.

5. _, On a theorem from linear algebra, Izv. Akad. Nauk Moldav. SSR Ser. Fiz.-Tekn. Mat. Nauk 1982, no. 3, 29-33.

6. W. Stoll, The Ahlfors-Weyl theory of meromorphic maps on parabolic manifolds, Lecture Notes in Math., vol. 981, Springer-Verlag, 1983, pp. 101-219.

7. __ Value distribution theory of meromorphic maps, Aspects of Mathematics, E7, Vieweg, 1985.

Department of Mathematics, University of Notre Dame, Notre Dame, Indiana 46556

Current address: Department of Mathematics, Moorhead State University, Moorhead, Minnesota 56560 